\title{
Socio-economic status, place of residence and dietary habits among the elderly: the Mediterranean islands study
}

\author{
Alexia Katsarou ${ }^{1}$, Stefanos Tyrovolas ${ }^{1}$, Theodora Psaltopoulou ${ }^{2}$, Akis Zeimbekis ${ }^{3}$, \\ Nikos Tsakountakis ${ }^{4}$, Vassiliki Bountziouka ${ }^{1}$, Efthimios Gotsis ${ }^{1}$, George Metallinos ${ }^{1}$, \\ Evangelos Polychronopoulos ${ }^{1}$, Christos Lionis $^{4}$ and Demosthenes Panagiotakos ${ }^{1, *}$ \\ 'Department of Nutrition Science - Dietetics, Harokopio University, 46 Paleon Polemiston St., Glyfada, 16674 , \\ Attica, Athens, Greece: ${ }^{2}$ Department of Hygiene, Epidemiology, and Medical Statistics, School of Medicine, \\ University of Athens, Athens, Greece: ${ }^{3}$ Health Center of Kalloni, General Hospital of Mitilini, Mitilini, Greece: \\ ${ }^{4}$ Clinic of Social and Family Medicine, School of Medicine, University of Crete, Heraklion, Greece
}

Submitted 19 August 2009: Accepted 4 February 2010: First published online 31 March 2010

\begin{abstract}
Objective: To investigate whether the socio-economic status (SES) of elderly eastern Mediterranean islanders is associated with their dietary habits, particularly with adherence to the traditional Mediterranean diet.

Design: Cross-sectional.

Setting: Adherence to the Mediterranean diet was measured by the MedDietScore (range: 0-55), whereas SES was estimated using education and financial status.

Subjects: During 2005-2007, 300 men and women from Cyprus, 100 from Samothraki, 142 from Mitilini, 114 from Kefalonia, 131 from Crete, 150 from Lemnos, 150 from Corfu and 103 from Zakynthos (aged 65-100 years), free of known chronic diseases, participated in the survey.

Results: Multiple linear regression analysis revealed that belonging to the highest SES was associated with a higher MedDietScore $(P<0 \cdot 01)$, after adjusting for potential sociodemographic, lifestyle, dietary and clinical confounders. A significant positive association was also found between MedDietScore and years of school $(P=0 \cdot 004)$, as well as financial status $(P=0 \cdot 001)$.

Conclusions: Older Greek people of higher SES seem to follow a relatively healthier diet. Both education and income seem to play a role in this issue. Thus, public health policy makers should focus on people with low SES in order to improve their quality of diet and, consequently, their health status.
\end{abstract}

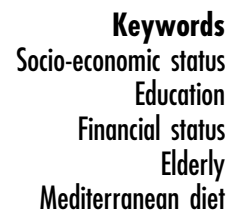

Socio-economic status (SES), as defined by occupation, education and income, has long been associated with different morbidity and mortality rates across strata from certain diseases, mainly chronic ones ${ }^{(1-3)}$. It seems that there is a gradient between SES and health, in which the more socio-economically benefited a person is, the better their health status ${ }^{(4)}$. Moreover, there is evidence indicating that the relationship between SES and health seems to influence all levels of the socio-economic hier$\operatorname{archy}^{(1,5)}$. In Westernised countries especially, CVD has been found to be more common in lower SES groups after the middle of the 20th century, and this trend is also evident in obesity, diabetes, stroke, metabolic syndrome, osteoporosis and some forms of cancer ${ }^{(6-8)}$.

Dietary recommendations and guidelines have been formulated to preserve and promote individual and public health ${ }^{(44)}$. High-energy and low-quality diets have been linked to increased morbidity and mortality, in contrast with healthy dietary patterns that promote longevity and decrease the risk for CVD and some types of cancers ${ }^{(9-11)}$. There is growing evidence that the Mediterranean diet consists of a dietary pattern that promotes longevity and reduces the risk for all-cause mortality ${ }^{(12-15)}$. At this point it should be mentioned that the majority of studies in the nutritional field have followed the approach of assessing single food items or nutrients, instead of dietary patterns (like the mediterranean diet) ${ }^{(16,17)}$. However, people do not eat isolated nutrients, but rather consume meals consisting of a variety of foods with complex combinations of nutrients. This is the reason why assessing dietary patterns, the high inter-correlation of nutrients and foods, should be captured, and the complex interactive effects of many dietary exposures be controlled ${ }^{(16,18)}$. Some research has also been conducted concerning the adoption of a healthy lifestyle, such as the Mediterranean dietary pattern, among the elderly, a rapidly growing segment of the population. It seems that the traditional Mediterranean diet is positively associated with longevity among the elderly ${ }^{(19)}$. In addition, a greater adherence to a plantbased diet among the elderly is found to be associated with lower all-cause mortality ${ }^{(20)}$, whereas it has been shown that 
the adoption of healthy diets reduces risk factors for CVD and certain types of cancer, and increases quality of life ${ }^{(21)}$.

Several studies have shown that diet quality seems to depend on socio-economic variables, such as occupation, income and education ${ }^{(21-26)}$. In developed countries, there is plenty of literature suggesting that, traditionally, lower socio-economic levels are characterised by unhealthier dietary habits, compared to upper socio-economic levels ${ }^{(24-26)}$. Moreover, some studies in the Mediterranean area have shown that people in low SES groups showed lower adherence to the Mediterranean diet ${ }^{(24)}$, whereas other studies showed the opposite ${ }^{(23)}$. According to current evidence, higher SES groups are characterised by higher consumption of non-refined grains, lean meat, fish, dairy products, fruit and vegetables compared to lower SES groups ${ }^{(25)}$, but there is still a considerable lack of evidence regarding this issue in various parts of the world, and particularly in the elderly ${ }^{(27,28)}$.

Elderly men and women from various Eastern Mediterranean islands ${ }^{(28)}$ were thus enrolled in the Mediterranean islands (MEDIS) study, which investigated whether diet quality, as measured by adherence to the traditional Mediterranean diet, was associated with SES of the participants.

\section{Materials and methods}

\section{Participants}

The MEDIS study ${ }^{(29)}$ is a health and nutrition survey that was designed to evaluate bio-clinical, lifestyle, behavioural and dietary characteristics of elderly people living on the Eastern Mediterranean islands. A random, populationbased, multi-stage sampling method (i.e. three age group levels (65-75, 75-85 and 85 and above years) and two sex levels) was used to select men and women from Cyprus Republic and Mitilini, Samothraki, Cephalonia, Crete, Corfu, Lemnos and Zakynthos islands in Greece. Individuals residing in assisted-living centres, as well as those with a clinical history of CVD or cancer, were not included in the survey. The target sample group size was 300 people from Cyprus and 150 from each one of the other islands. Of the initial sample, 553 men (mean age 76 (SD 7) years) and 637 women (mean age 74 (SD 7) years; $n$ 1190) agreed to participate (Cyprus, $n$ 300; Mitilini, $n$ 142; Samothraki, $n$ 100; Cephalonia, $n 115$; Crete, $n 131$; Corfu, $n 149$; Lemnos $n$ 150; and Zakinthos, $n$ 103). Four hundred and sixty (39\%) people were living in rural areas of the islands. The participation rate varied between $75 \%$ and $89 \%$, on different islands. A group of health scientists (i.e. physicians, dietitians and nurses) with experience in field investigation collected all the required information, using a quantitative questionnaire and standard procedures.

\section{Bioethics}

The retrieved data were confidential, and the study followed the ethical considerations provided by the World
Medical Association (52nd WMA General Assembly, Edinburgh, Scotland, October 2000). Moreover, the Institutional Review Board approved the design, procedures and aims of the study. Before the interviews, participants were informed about the aims and procedures of the study and provided their consent.

\section{Assessment of socio-economic status}

SES was estimated on the basis of years of education and the mean annual income of the family (through selfreports and divided into four classes: low $(<€ 7200)$, medium (€7200-12000), good ( $>€ 12000-20000)$ and very good $(>€ 20000)$ ) over the past 3 years. It should be noted that given that most of the elderly who participated in our survey were pensioners, there was little benefit in the inclusion of occupation in the SES calculation. Taking into consideration people within the family who were not working, we used the average family income, whereas for unemployed individuals we used the basic monthly allowance provided by the Social Service Office. As no accurate measure of SES exists in Greece, a special 'socioeconomic' index was developed in order to evaluate the SES of the participants. Thus, the socio-economic index was developed as follows. We multiplied the years in school for each individual with the classification of their annual income (range of the index: 0-64). This index was normally distributed according to the KolmogorovSmirnov criterion. We then divided our sample into three equally sized groups (tertiles) according to this score; the first tertile (score: <4) was termed 'low SES', the second tertile was 'medium SES' (score: 4-10) and the third tertile was 'high SES' (score: >10). This type of assessment of SES has already been used in the literature ${ }^{(30)}$ and it is not unusual in the quantification of SES for only one or two of its components (education, income and occupation) to be combined $^{(31,32)}$. Of course, education level and financial status in multi-adjusted models could also be independently used. However, this would raise the issue of colinearity, which influences the robustness of the estimates of the models.

\section{Measurements}

The retrieved information also included basic demographic characteristics, such as age (in years), sex, whether cohabiting and place of living, as well as various clinical and lifestyle characteristics. Diabetes mellitus (type 2) was determined by fasting plasma glucose levels $>125 \mathrm{mg} / \mathrm{dl}$ or use of special antidiabetic medication; participants who had blood pressure levels $\geq 140 / 90 \mathrm{mmHg}$ or used antihypertensive medications were classified as hypertensive. Fasting blood lipid levels were also recorded and hypercholesterolaemia was defined as total serum cholesterol levels $>200 \mathrm{mg} / \mathrm{dl}$ or the use of lipid-lowering agents. Body height and weight were measured and obesity was defined as BMI $>29.9 \mathrm{~kg} / \mathrm{m}^{2}$. Current smokers were defined as those who smoked at least one cigarette per day or had 
stopped smoking over the past 12 months. The remaining participants were defined as rare or non-current smokers. Dietary habits were assessed through a semi-quantitative, validated (in our Institution) FFQ, which included fifty-nine basic food items and behaviours. Consumption of various food groups and beverages (i.e. meat and products, fish and fish products, milk and other dairy products, fruit, vegetables, greens and salads, legumes, cereals, olive oil, coffee and tea and soft drinks) was assessed by times of serving consumed during a regular week or month during the previous year. Consumption of alcoholic beverages (i.e. wine, beer, etc.) was measured in terms of wineglasses adjusted for ethanol intake (e.g. one $100 \mathrm{ml}$ glass of wine was considered to have $12 \%$ ethanol). To evaluate overall dietary habits, a special diet score, the MedDietScore (range: $0-55)^{(18)}$, was used to assess adherence to this traditional dietary pattern. For the consumption of foods presumed to be close to this pattern (i.e. those suggested on a daily basis or more than four servings per week), scores of 0 and 5 were assigned when a participant reported no consumption and daily consumption, respectively. On the other hand, for the consumption of foods presumed to not adhere to this diet (meat and meat products) the opposite scores were assigned (i.e. 0 when a participant reported almost daily consumption and 5 for rare or no consumption). With regard to the alcohol intake, a score of 5 was assigned for consumption of less than three wineglasses per day, a score of 0 for none or consumption of more than seven wineglasses per day and scores of 4, 3, 2 and 1 for the consumption of three, four to five, six and seven wineglasses, respectively. Higher values of this diet score indicate greater adherence to the Mediterranean diet. The level of adherence to the Mediterranean diet was evaluated as the ratio of the mean diet score divided by 55 , which is the total possible achievable score (i.e. MedDietScore/55×100\%). Higher values of this diet score indicate greater adherence to the Mediterranean diet. Physical activity was evaluated by using the shortened version of the self-report and the International Physical Activity Questionnaire for the elderly ${ }^{(33)}$. Participants who did not report any physical activity were defined as sedentary (64\%).

Details about the aims and procedures of the MEDIS study have been presented elsewhere ${ }^{(29)}$.

\section{Statistical analysis}

Continuous variables are presented as mean and SD values, whereas categorical variables are presented as relative frequencies. Associations between categorical variables were tested by the calculation of $\chi^{2}$ test, whereas differences between SES groups and several participants' characteristics were tested by the use of ANOVA, for the normally distributed variables. Spearman's correlation coefficient was used to examine the association among years of school, financial status and MedDietScore levels. Furthermore, multiple linear regression models were used to evaluate the association of
MedDietScore with SES classes after adjusting for potential confounders. Normality was tested by using the Shapiro-Wilk test and P-P plots. All reported $P$ values are based on two-sided tests and compared with a significance level of $5 \%$. However, when multiple comparisons were made within a test, we used the Bonferroni correction in order to account for the increase in type I error. The Statistical Package for the Social Sciences statistical software package version 14 (SPSS Inc., Chicago, IL, USA) was used for all statistical calculations.

\section{Results}

Table 1 illustrates the distributions of sociodemographic and lifestyle characteristics by SES group. There is a variation in age among the three SES groups $(P<0 \cdot 001)$, with the younger individuals of the sample at the higher SES level. As expected, the proportion of men was lower in the low SES group than in the medium or higher SES group $(P<0 \cdot 001)$. Moreover, more individuals in the high SES group lived in rural areas than those in the other groups $(P<0 \cdot 001)$; the latter could confound the main relationship between SES and Mediterranean diet adherence, and will be taken into account in the following analyses. With regard to living conditions, people in the lowest SES group were more likely to live alone $(P=0 \cdot 036)$ and to have higher BMI and lower physical activity levels than those in the highest SES group $(P<0 \cdot 001)$. In addition, a positive association among MedDietScore, years of school $(r=0.08, P=0.004)$ and financial status (low-moderate financial status $v$. good-very good: mean 33 (SD 4) $v$. mean 35 (SD 5), $P=0 \cdot 002$ ) was also observed.

The specific dietary habits of the participants in relation to their SES are presented in Table 2. As can be seen, people in the highest SES group consumed more quantities of fish and vegetables than those in the lowest SES group. No other significant differences were observed between social classes with regard to the specific eating habits of the participants. The results remained similar when the analysis was stratified by sex and place of residence (data not shown in text). With regard to the MedDietScore that assesses overall diet, elderly people in the highest SES group seemed to adhere more closely to the traditional Mediterranean diet than the other SES categories $(P=0 \cdot 025)$. However, the mean differences between SES groups in the MedDietScore are relatively small, achieving significance mainly due to the large sample and the small within-group variation. Nevertheless, it should be noted that all participants showed moderate adherence to the Mediterranean diet (i.e. the MedDietScore was $33 / 55$ or $60 \%$ of the total theoretical score in low SES people $v .33 / 55$ or $60 \% v .34 / 55$ or $62 \%$, in medium and high SES people, respectively).

We have already observed a positive relationship between greater adherence to the traditional Mediterranean 
Table 1 Sociodemographic and lifestyle characteristics of elderly men and women participated in the Mediterranean Islands study, by socio-economic status groupt

\begin{tabular}{|c|c|c|c|c|c|c|c|c|c|}
\hline & \multicolumn{8}{|c|}{ Socio-economic status group } & \multirow[b]{3}{*}{$P \ddagger$} \\
\hline & \multicolumn{2}{|c|}{ Low (n 654) } & \multicolumn{2}{|c|}{ Medium ( $n$ 254) } & \multicolumn{2}{|c|}{ High ( $n$ 221) } & \multicolumn{2}{|c|}{ Total $(n 1129)$} & \\
\hline & Mean or $\%$ & SD & Mean or $\%$ & SD & Mean or $\%$ & SD & Mean or $\%$ & $\overline{S D}$ & \\
\hline Age (years) & 75 & $7 \cdot 1$ & 73 & $6 \cdot 9$ & 72 & $6 \cdot 5^{*}$ & 74 & $7 \cdot 0$ & $<0.001$ \\
\hline Sex (male, \%) & 38 & & $59^{\star *}$ & & $58^{\star \star}$ & & 47 & & $<0.001$ \\
\hline Living in urban areas (\%) & 68 & & 62 & & $43^{\star \star}$ & & 62 & & $<0.0001$ \\
\hline Living alone (\%) & 31 & & $24^{*}$ & & $24^{*}$ & & 28 & & 0.036 \\
\hline Smoking current (\%) & 11 & & 18 & & 18 & & 14 & & 0.006 \\
\hline Physical activity (\%) & 32 & & 33 & & $51^{\star *}$ & & 36 & & $<0.001$ \\
\hline $\mathrm{BMI}\left(\mathrm{kg} / \mathrm{m}^{2}\right)$ & $29 \cdot 1$ & $5 \cdot 0$ & $28 \cdot 2$ & $4 \cdot 8^{*}$ & $28 \cdot 1$ & $4 \cdot 5^{\star}$ & $28 \cdot 6$ & $4 \cdot 8$ & $<0.001$ \\
\hline Hypertension (\%) & 70 & & 66 & & $61^{*}$ & & 67 & & 0.03 \\
\hline Hypercholesterolaemia (\%) & 58 & & 53 & & $48^{*}$ & & 55 & & 0.02 \\
\hline Diabetes (\%) & 22 & & 24 & & 17 & & 21 & & $0 \cdot 18$ \\
\hline
\end{tabular}

tData are presented as mean (SD) or relative frequencies (\%).

$\ddagger$ All $P$ values $(P<0.05$ and $P<0.01)$ between high, medium $v$. low socio-economic groups, after correcting for multiple comparisons using the Bonferroni rule. ${ }^{*} P$ value $<0.05$ between high and low socio-economic groups.

${ }^{* *} P$ value $<0.001$ between high and low socio-economic groups.

Table 2 Dietary characteristics (servings/week) of elderly men and women participated in the Mediterranean Islands study, by socioeconomic status group

\begin{tabular}{|c|c|c|c|c|c|c|c|}
\hline & \multicolumn{6}{|c|}{ Socio-economic status group } & \multirow[b]{3}{*}{$P^{*}$} \\
\hline & \multicolumn{2}{|c|}{ Low ( $n$ 654) } & \multicolumn{2}{|c|}{ Medium ( $n$ 254) } & \multicolumn{2}{|c|}{ High (n 221) } & \\
\hline & Mean & SD & Mean & SD & Mean & SD & \\
\hline Meat (red or white) and products & $2 \cdot 7$ & $1 \cdot 1$ & $2 \cdot 8$ & $1 \cdot 1$ & $2 \cdot 6$ & $1 \cdot 0$ & 0.51 \\
\hline Fish & $2 \cdot 0$ & $1 \cdot 1$ & $2 \cdot 0$ & $1 \cdot 2$ & $2 \cdot 4$ & $1 \cdot 1^{*}$ & 0.02 \\
\hline Vegetables & 53 & 36 & 61 & $35^{*}$ & 58 & $35^{\star}$ & 0.01 \\
\hline Fruits & 20 & $6 \cdot 4$ & 18 & $6 \cdot 8$ & 20 & $6 \cdot 7$ & 0.24 \\
\hline Legumes & $2 \cdot 7$ & $1 \cdot 2$ & $2 \cdot 8$ & $1 \cdot 2$ & $2 \cdot 8$ & $1 \cdot 2$ & 0.43 \\
\hline Cereals & 27 & 14 & 26 & 14 & 28 & 13 & 0.55 \\
\hline Olive oil use (times/week) & $5 \cdot 2$ & 1.6 & $4 \cdot 8$ & 1.9 & 4.9 & $1 \cdot 9$ & $0 \cdot 10$ \\
\hline Alcohol drinking (glasses/d) & $1 \cdot 0$ & 0.9 & $1 \cdot 0$ & $1 \cdot 0$ & 0.9 & $1 \cdot 0$ & 0.89 \\
\hline MedDietScore (range: 0-55) & 33.5 & $3 \cdot 4$ & $33 \cdot 2$ & $4 \cdot 5$ & $34 \cdot 2$ & $4 \cdot 5^{\star}$ & 0.02 \\
\hline
\end{tabular}

*All $P$ values $(P<0.05$ and $P<0.01)$ between high, medium $v$. low socio-economic groups, after correcting for multiple comparisons using the Bonferroni rule.

diet and higher SES. However, due to residual confounding, we also applied multi-adjusted analysis. A positive relationship between the adoption of the Mediterranean diet and SES was revealed (Table 3), after age and sex adjustments (model 1). We then adjusted for multiple confounders, such as place of residence, whether cohabiting, BMI (models 2 and 3), as well as physical activity, current smoking (model 4), presence of diabetes, hypertension and hypercholesterolaemia (model 5). People in the highest tertile of the SES had higher MedDietScore results (i.e. positive $\beta$-coefficient in all multi-adjusted models). The latter observation with regard to the effect of SES on dietary habits lost its significance $(P=0 \cdot 08)$ when the presence and management of clinical conditions such as hypertension, hypercholesterolaemia and diabetes were taken into account (model 5). It is worthy of note that the significance of the association between the place of residence and the MedDietScore (i.e. people from rural areas adhered more closely to the traditional dietary habits than those living in urban areas) remained high after all adjustments were made $(P<0 \cdot 001)$. Moreover, current smoking habits were associated with lower levels of adherence to the traditional Mediterranean diet, providing a more unhealthy profile of the participants (model 5).

\section{Discussion}

We found among elderly people living on the Eastern Mediterranean islands that lower SES, smoking habits and urban place of residence are factors that are associated with lower adherence to a healthy dietary pattern, in the present case the traditional Mediterranean dietary pattern. It is of interest that specific food habits did not differ between SES classes, with the exception of fish and vegetable consumption, although the overall dietary habits seem to be influenced by the participants' SES (Table 2). However, it should be noted that the previous relationship between SES and dietary habits was mediated by the presence and management of classic CVD risk factors such as hypertension, hypercholesterolaemia and diabetes (Table 3). 
Most studies that have evaluated the associations of dietary habits with human health have followed the classic approach of assessing single food items or nutrients, instead of using a holistic dietary approach that assesses dietary patterns. Although this type of analysis is quite valuable in assessing the effect of specific foods and nutrients on chronic diseases, it has several conceptual and methodological limitations, e.g. the consumption of various foods clusters together (i.e. vegetables, greens, fruits, etc.), while other food options seem to have inverse relationships (i.e. increased meat intake is correlated with reduced fish consumption). Therefore, the 'single nutrient' approach may be inadequate for taking into account interactions among foods and nutrients, in studies of free-living people. Thus, a holistic dietary approach to disease prevention using dietary patterns is strongly recommended, as patterns capture the extremes of dietary habits and pre-empt nutritional confounding, possibly affect modification among nutritional variables through the same procedure, and do not tend to be biased $^{(16-18)}$. In addition, studying dietary patterns could have important public health implications, as the holistic dietary approach might be easy for the public to translate into diets. In the present study, the holistic dietary approach was followed to evaluate the influence of SES on peoples' dietary habits, revealing interesting associations.

Eliminating health disparities is one of the major goals of the Healthy People 2010 public agenda ${ }^{(34)}$ and for many other public health policies as well, with health disparities of the elderly, due to their social class, constituting a significant public health subject. We can assume that the lower rate of adoption of the Mediterranean diet observed in the low and medium SES (Table 2) groups is possibly attributed to the poor purchasing power of the elderly, with regard to the consumption of fruit, vegetables, legumes and fish prescribed by the traditional Mediterranean diet. In Mediterranean countries, this native plant-based diet is probably not considered a very expensive diet, mainly due to the fact that (i) in such regions as the Greek islands plants and fruits are widely available and (ii) people like to grow fruits and vegetables $^{(35)}$. First, in several studies, the highest diet costs were found to be associated with antioxidant phytonutrients found in fruits and vegetables ${ }^{(36)}$. In addition, lower energy density and higher nutritional-quality foods, such as those that characterise the traditional Mediterranean diet, have been found to be associated with higher energyadjusted diet costs ${ }^{(37)}$. However, we must underline the fact that the level of adherence to the Mediterranean dietary pattern seems to be moderate (i.e. approximately $60 \%$ of the best achievable score) in all SES groups. However, compared with earlier studies, our results show that elderly people living on the Greek islands and Cyprus had a higher level of adherence than middle-aged adults (mean age 50 years) living in the Athens ${ }^{(35)}$. Furthermore, another large-scale initiative, the HALE project (Healthy Ageing: a 
Longitudinal Study in Europe), which included elderly people of the same age range as in our sample, showed an average $50 \%$ level of adherence to the Mediterranean diet $^{(38)}$. Thus, based on earlier studies, it seems that elderly people living on the Greek islands, whether grouped in low, medium or high SES, seem to follow these traditional dietary habits more closely than Greek middle-aged people in the same SES group or elderly people from other countries.

Second, there is some evidence associating dietary habits with educational status and thus social class, according to which low educational status has been found to be associated with lower consumption of fresh fruit and vegetables, and unhealthy dietary habits with lack of knowledge regarding the effect of healthy eating on health and well-being ${ }^{(21,39)}$. However, there is evidence supporting that in Southern European countries, high education status people tend to consume more fresh fruit and vegetables compared with the low educated ${ }^{(40)}$. In addition, there is a great deal of literature suggesting that, traditionally, lower socio-economic levels are characterised by unhealthier dietary habits compared with upper socio-economic levels ${ }^{(23,24,41,42)}$. In addition, several studies in the Mediterranean area have shown that people with low SES had lower adherence to the Mediterranean diet ${ }^{(23-26)}$, as in the present study. Moreover, it has been suggested that low educational status can partially explain the lower adherence to the Mediterranean diet by the elderly ${ }^{(23-26)}$. Nutrition and dietary habits have a complex relationship with cognition or learning, and among educational parameters influencing unhealthy dietary habits even in the elderly could be the lack of nutritional knowledge and the fact that they are not interested in following proper nutritional prevention messages, lack of cooking skills and lack of interest in cooking, lack of motivation and an erroneous perception of body weight ${ }^{(21)}$. Thus, it is not strange that in lower SES group there are higher rates of obesity and metabolic syndrome. The latter could also be one of the reasons that the effect of SES on dietary habits slightly lost its significance when presence and management of clinical conditions, like hypertension, hypercholesterolaemia and diabetes, were taken into account. Moreover, this mediating effect could also attribute to the reason that elderly people may give more emphasis to their medical treatment including dietary recommendations.

A third parameter of SES is occupation, but when concerning older individuals, we should refer to their occupational skills. Therefore, we can assume that lower SES is associated with poor occupational skills. In the present study, we have not attempted to consider occupational skills because in our data set almost all of the elderly participants were retired.

The existence of behavioural risk factors, i.e. lower adherence to the traditional Mediterranean diet and lower rates of physical activity in the socially disadvantaged elderly in our sample, may raise the probability of major health problems, such as cardiovascular outcomes and cancer. Therefore, SES could be a 'fundamental cause' of mortality disparities in the elderly because difference in SES embodies a gradient in factors, such as money, knowledge, prestige, power and beneficial social connections that protect or deteriorate health ${ }^{(43-45)}$.

\section{Strengths and limitations}

The present study is one of the first studies evaluating a large sample of 'healthy', free-living elderly people living on the Eastern Mediterranean islands. These people belong to the generation of individuals in whom the traditional Mediterranean diet first originated. It is notable that individuals from two of these islands (i.e. Crete and Corfu) participated in the Seven Countries Study in the late 1950s, which revealed, among other findings, the protective role of the Mediterranean type of diet on CVD. Since then these islands have rarely been studied.

However, the present study has some limitations, mainly in its cross-sectional design, and thus the results cannot be interpreted causally. Moreover, the findings may have been influenced by potential recall bias, particularly during the assessment of dietary habits, because the participants' reports could be influenced by memory problems, their low SES (as there is a possibility of overestimation to the better from people belonging into low SES groups), as well as the fact that diet evaluation took place only once. In addition, we cannot generalise our findings among other elderly populations.

\section{Conclusions}

The initial observation that low and medium SES is associated with lower adherence to the Mediterranean diet seems to be explained by the variation in the presence of common clinical conditions prevailing in the elderly, such as hypertension, hypercholesterolaemia and diabetes. Public health policies implemented by regional authorities and governmental agencies should focus on elderly populations, especially those of low or medium SES.

\section{Acknowledgements}

The study is funded by research grants from the Hellenic Heart Foundation. There are no conflicts of interest. A.K. and S.T. wrote the paper and performed the data analyses. D.P. designed and supervised the study, supervised the data analyses and reviewed the paper, A.Z., N.T., V.B., E.G., G.M., E.P. and C.L. participated in the sampling and critically reviewed the paper, and T.P. critically reviewed the paper. The authors particularly, grateful to the men and women from the islands of Cyprus, Mitilini, Samothraki, Crete, Corfu, Lemnos, Zakynthos and Cephalonia, who participated in and collaborated in the present study. The authors also 
thank M. Tornaritis, A. Polystipioti, M. Economou, (field investigators from Cyprus), K. Gelastopoulou, I. Vlachou (field investigator from Mitilini), I. Tsiligianni, M. Antonopoulou, N. Tsakountakis, K. Makri (field investigators from Crete), E. Niforatou, V. Alpentzou, M. Voutsadaki, M. Galiatsatos (field investigators from Cephalonia), K. Voutsa, E. Lioliou, M. Miheli (field investigator from Corfu), G. Pounis, S. Tyrovolas, E. Papavenetiou, E. Apostolidou, G. Papavassiliou and P. Stravopodis (field investigators from Zakynthos) for their substantial assistance in the enrollment of the participants.

\section{References}

1. Marmot MG, Shipley MJ \& Rose G (1984) Inequalities in death: specific explanations of a general pattern? Lancet $\mathbf{1}$, 1003-1006.

2. Smith GD \& Egger M (1993) Socioeconomic differentials in wealth and health. BMJ 307, 1085-1086.

3. Adler NE, Boyce T, Chesney MA et al. (1994) Socioeconomic status and health: the challenge of the gradient. American Psychologist 49, 15-24.

4. Adler ED \& Ostrove MJ (1993) Socioeconomic status and health: what we know and what we don't. Ann N Y Acad Sci 896, 3-15.

5. Marmot MG, Smith GD, Stansfeld S et al. (1991) Health inequalities among British civil servants: the Whitehall II study. Lancet 337, 1387-1393.

6. Panagiotakos DB, Lionis C, Anastasiou F et al. (2008) Prevalence of risk factors for cardiovascular disease by socio-economic status among the elderly from Mediterranean islands: the MEDIS study. CVD Prev Control 3, 153-162.

7. Nordstrom KC, Diez Roux VA, Jackson AS et al. (2004) The association of personal and neighbourhood socioeconomic indicators with subclinical cardiovascular disease in an elderly cohort. The cardiovascular health study. Soc Sci Med 59, 2139-2147.

8. Melchior M, Goldberg M, Krieger N et al. (2005) Occupational class, occupational mobility and cancer incidence among middle-aged men and women: a prospective study of the French GAZEL cohort. Cancer Causes Control 16, 515-524.

9. Nishida C, Uauy R, Kumanyika S et al. (2004) The joint WHO/FAO expert consultation on diet, nutrition and the prevention of chronic diseases: process, product and policy implications. Public Health Nutr 7, 245-250.

10. Panagiota NM, Kipnis V, Anne CM et al. (2007) Mediterranean dietary pattern and prediction of all-cause mortality in a US population. Arch Intern Med 6, 72461-72468.

11. Mantzoros CS, Frank B, Heidemann HC et al. (2008) Dietary patterns and risk of mortality from cardiovascular disease, cancer and all causes in a prospective cohort of women. Circulation 118, 230-237.

12. Trichopoulou A \& Critselis E (2004) Mediterranean diet and longevity. Eur J Cancer Prev 13, 453-456.

13. Chrysohoou C, Panagiotakos DB, Pitsavos C et al. (2004) Adherence to the Mediterranean diet attenuates inflammation and coagulation process in healthy adults: the ATTICA study. J Am Coll Cardiol 44, 152-158.

14. Willett WC, Sacks F, Trichopoulou A et al. (1995) Mediterranean diet pyramid: a cultural model for healthy eating. Am J Clin Nutr 61, 1402-1406.

15. Sofi F, Cesari F, Abbate R et al. (2008) Adherence to Mediterranean diet and health status: meta-analysis. BMJ 337, a1344.
16. Hu FB (2002) Dietary pattern analysis: a new direction in nutritional epidemiology. Curr Opin Lipidol 13, 3-9.

17. Kant AK (1996) Indexes of overall diet quality: a review. J Am Diet Assoc 96, 785-791.

18. Panagiotakos DB, Pitsavos C \& Stefanadis C (2006) Dietary patterns: a Mediterranean diet score and its relation to CVD risk and markers. Nutr Metab Cardiovasc Dis 16, 559-568.

19. Trichopoulou A (2004) Traditional Mediterranean diet and longevity in the elderly: a review. Public Health Nutr 7 , 943-947.

20. Bamia C, Trichopoulos D, Ferrari P et al. (2007) Dietary patterns and survival of older Europeans: The EPIC-Elderly Study (European Prospective Investigation into Cancer and Nutrition). Public Health Nutr 10, 590-598.

21. Darmon N \& Drewnowski A (2008) Does social class predict diet quality? Am J Clin Nutr 87, 1107-1117.

22. Krieger N, Williams DR \& Moss NE (1997) Measuring social class in US public health research: concepts, methodologies, and guidelines. Annu Rev Public Health 18, 341-378.

23. Scali J, Richard A \& Gerber M (2001) Diet profiles in a population sample from Mediterranean southern France. Public Health Nutr 4, 173-182.

24. Pitsavos CE, Panagiotakos DB, Chrysohoou CA et al. (2002) Education and acute coronary syndromes: results from the CARDIO2000 epidemiological study. Bull World Health Organ 80, 371-377.

25. Vlismas K, Stavrinos V \& Panagiotakos DB (2009) Socioeconomic status, dietary habits and health-related outcomes in various parts of the world: a review. Cent EurJ Public Health 17, 55-63.

26. Mishra G, Ball K, Arbuckle J \& Crawford D (2002) Dietary patterns of Australian adults and their association with socioeconomic status: results from the 1995 National Nutrition Survey. Eur J Clin Nutr 56, 687-693.

27. Windham CT, Wyse BW, Hansen RG et al. (1983) Nutrient density of diets in the USDA Nationwide Food Consumption Survey, 1977-1978: I. Impact of socioeconomic status on dietary density. J Am Diet Assoc 82, 28-34.

28. Panagiotakos D, Bountziouka V, Zeimbekis A et al. (2007) Food pattern analysis and prevalence of cardiovascular disease risk factors among elderly people from Mediterranean islands. J Med Food 10, 615-621.

29. Tyrovolas S, Lionis C, Zeimbekis A et al. (2009) Increased body mass and depressive symptomatology are associated with hypercholesterolaemia, among elderly individuals; results from the MEDIS study. Lipids Health Dis $\mathbf{8}, 10$.

30. Manios Y, Panagiotakos DB, Pitsavos C et al. (2005) Implication of socio-economic status on the prevalence of overweight and obesity in Greek adults: the ATTICA study. Health Policy 74, 224-232.

31. Lidfeldt J, Li T, Hu F et al. (2007) A prospective study of childhood and adult socioeconomic status and incidence of type 2 diabetes in women. Am J Epidemiol 165, 882-889.

32. Avendano M, Kawachi I, Van Lenthe F et al. (2006) Socioeconomic factors and stroke incidence in the US elderly: the role of risk factors in the EPESE Study. Stroke 37, 1368-1373.

33. Craig CL, Marshall AL \& Sjostrom M (2003) International physical activity questionnaire: 12-country reliability and validity. Med Sci Sports Exerc 35, 1381-1395.

34. Mensah GA (2005) Eliminating disparities in cardiovascular health: six strategic imperatives and a framework for action. Circulation 111, 1332-1336.

35. Panagiotakos DB, Pitsavos C, Chrysohoou C et al. (2008) Dietary habits mediate the relationship between socioeconomic status and CVD factors among healthy adults: the ATTICA study. Public Health Nutr 11, 1342-1349.

36. Andrieu E, Darmon N \& Drewnowski A (2006) Low-cost diets: more energy, fewer nutrients. Eur J Clin Nutr 60, 434-436. 
37. Maillot M, Darmon N, Vieux F et al. (2007) Low energy density and higher nutritional quality are each associated with higher diet costs in French adults. Am J Clin Nutr 86, 690-696.

38. Knoops KT, de Groot LC, Kromhout D et al. (2004) Mediterranean diet, lifestyle factors, and 10-year mortality in elderly European men and women. The HALE project. JAMA 292, 1433-1439.

39. Roos G, Johansson L, Kasmel A et al. (2000) Disparities in vegetable and fruit consumption: European cases from the North to the South. Public Health Nutr 4, 35-43.

40. Irala-Estevez JD, Groth M, Johansson L et al. (2000) A systematic review of socioeconomic differences in food habits in Europe: consumption of fruit and vegetables. Eur J Clin Nutr 54, 706-714.

41. Trichopoulou A, Naska A \& Costacou T (2002) Disparities in food habits across Europe. Proc Nutr Soc 61, 553-558.
42. Caraher M, Dixon P, Lang T et al. (1998) Access to healthy foods: part I. Barriers to accessing healthy food: differentials by gender, social class, income and mode of transport. Health Educ J 57, 191-201.

43. Phelan JC, Link BG, Diez-Roux A et al. (2004) Fundamental causes of social inequalities in mortality: a test of the theory. J Health Soc Behav 45, 265-285.

44. Kfam H, Brussaard JH, Kruizinga AG et al. (2003) Socioeconomic status, dietary intake and $10 \mathrm{y}$ trends: the Dutch National Food Consumption Survey. Eur J Clin Nutr 57, 128-137.

45. Loucks EB, Rehkopf DH, Thurston RC et al. (2007) Socioeconomic disparities in metabolic syndrome differ by gender: evidence from NHANES III. Ann Epidemiol 17, 19-26. 